\title{
Evolution of a Binary Black Hole with a Retrograde Circumbinary Accretion Disk
}

\author{
Jeremy D. Schnittman \\ NASA Goddard Space Flight Center, Greenbelt, MD 20771 \\ Joint Space-Science Institute, College Park, MD 20742 \\ Julian H. Krolik \\ Department of Physics and Astronomy, Johns Hopkins University, 3400 N Charles Street, \\ Baltimore, MD 21218
}

\begin{abstract}
We consider the evolution of a supermassive black hole binary (SMBHB) surrounded by a retrograde accretion disk. Assuming the disk is exactly in the binary plane and transfers energy and angular momentum to the binary via direct gas accretion, we calculate the time evolution of the binary's semi-major axis $a$ and eccentricity $e$. Because the gas is predominantly transferred when the binary is at apocenter, we find the eccentricity grows rapidly while maintaining constant $a(1+e)$. After accreting only a fraction of the secondary's mass, the eccentricity grows to nearly unity; from then on, gravitational wave emission dominates the evolution, preserving constant $a(1-e)$. The high-eccentricity waveforms redistribute the peak gravitational wave power from the $\mathrm{nHz}$ to $\mu \mathrm{Hz}$ bands, substantially affecting the signal that might be detected with pulsar timing arrays. We also estimate the torque coupling binaries of arbitrary eccentricity with obliquely aligned circumbinary disks. If the outer edge of the disk is not an extremely large multiple of the binary separation, retrograde accretion can drive the binary into the gravitational wave-dominated state before these torques align the binary with the angular momentum of the mass supply.
\end{abstract}

\section{Introduction}

The orbital evolution of binary black holes has been a subject of considerable interest for more than thirty years, dating back to the work of Begelman et al. (1980). It has only grown in prominence in recent years, as it has become apparent both that most reasonablylarge galaxies contain supermassive black holes in their nuclei (Kormendy \& Richstone 1995; 
Magorrian et al. 1998) and that today's galaxies are the product of numerous mergers between smaller galaxies (Bell et al. 2006; McIntosh et al. 2008; de Ravel et al. 2009; Bridge et al. 2010), each of which likely contains its own nuclear black hole. As pointed out by Begelman et al. (1980), when two massive black holes in a galaxy approach near enough one another to form a gravitationally-bound binary, interactions with external stars become much less effective at bringing them still closer together. A number of processes have since been suggested as viable mechanisms to push such a system closer toward the merger of the two black holes themselves (Merritt \& Milosavljevió 2005).

A particularly popular such mechanism (Gould \& Rix 2000; Armitage \& Natarajan 2002; Escala et al. 2004, 2005; MacFadyen \& Milosavljević 2008; Cuadra et al. 2009) is accretion of gas onto the binary black hole through a circumbinary disk. In principle, the orbital plane of such a disk could be wholly independent of the orbital plane of the binary, particularly at large radius. However, a number of processes have been pointed out that tend to push the two orbital planes toward coincidence (Nixon 2012; Miller \& Krolik 2013). Whether the angular momenta of the two orbits align or counter-align, however, is less clear.

Retrograde circumbinary disks have certain special properties (Nixon et al. 2011; Roedig \& Sesana 2014; Bankert et al. 2014). Because the angular momentum acquired by accretion is directed opposite to the binary orbital angular momentum, accretion in such a context tends to drive a rapid decrease in the orbital angular momentum while only modestly decreasing the orbital energy. The result is an increase in the orbital eccentricity. Indeed, the first paper to consider evolution of this sort (Nixon et al. 2011) concluded on the basis of qualitative arguments that this eccentricity growth causes the pericenter distance $r_{p}=a(1-e)$ to become so small that gravitational wave $(\mathrm{GW})$ radiation rapidly drives the system to merger. Although GW radiation generically decreases eccentricity (Peters 1964), in their view the binary orbit would retain non-zero eccentricity all the way to merger.

Here we study this problem quantitatively. For a wide range of reasonable initial conditions and physical assumptions, we find the same robust result: rapid growth in eccentricity with constant apocenter $a(1+e)$ over a time long enough to accrete a mass comparable to that of the secondary (for equal-mass systems accreting at the Eddington limit this is a Salpeter time, $t_{\text {Sal }}=4.5 \times 10^{7}$ years, for a nominal radiative efficiency $\eta=0.1$ ). At that point the eccentricity reaches $e \gtrsim 0.99$, and gravitational wave evolution takes over. In this phase, GW losses occur almost entirely at pericenter, leading to evolution with constant $a(1-e)$ until the orbit is nearly circular at a binary separation of $\sim 100 r_{g}$ (the gravitational radius $\left.r_{g} \equiv G M / c^{2}\right)$.

This evolutionary behavior can have profound effects on pulsar timing array (PTA) observations of gravitational waves radiated by supermassive black hole mergers. If gas inflow 
in the center of a post-merger galaxy takes place in a stochastic manner, retrograde disks should be roughly as common as prograde disks, but much more efficient at effecting the binary merger. Rapid evolution from initial separations of $a \sim 10^{5} r_{g}$ down to $a \sim 10^{2} r_{g}$ will greatly reduce the number of systems potentially observable in this regime (Kocsis \& Sesana 2011; Sesana 2013; Ravi et al. 2014). As a result, the strength (Demorest et al. 2013) of the stochastic GW signal in the PTA band $f \sim 10^{-9}-10^{-8} \mathrm{~Hz}$ would be sharply diminished. In addition, the early onset of GW-dominated evolution could lead to a decoupling of the circumbinary disk from the inner binary at much greater radii than is typically assumed, altering electromagnetic signals associated with accretion onto the black holes (Milosavliević \& Phinnev 2005; Chang et al. 2010; Tanaka \& Menou 2010; Noble et al. 2012; Schnittman 2013, 2014; Farris et al. 2014b).

\section{Accretion-driven Evolution}

As shown in a companion paper describing magneto-hydrodynamic simulations of a retrograde circumbinary accretion disk (Bankert et al. 2014), the nearly axisymmetric density profile of the disk results in a very small gravitational torque on the binary. Thus, unlike the prograde case, the primary means of energy and angular momentum transfer from the disk to the binary is through direct accretion.

The eccentricity $e$ and semimajor axis $a$ of the binary can be written as

$$
e=\left[1+\frac{2 E J^{2}(1+q)^{6}}{G^{2} M^{5} q^{3}}\right]^{1 / 2}
$$

and

$$
a=-\frac{q}{(1+q)^{2}} \frac{G M^{2}}{2 E}
$$

with $M$ the binary's total mass, $J$ angular momentum, $E$ energy, and mass ratio $q \equiv$ $M_{2} / M_{1} l e 1$. The evolution equations are then given by

$$
\dot{e}=\frac{1-e^{2}}{2 e}\left[-2 \dot{J} / J-\dot{E} / E+5 \dot{M} / M+3 \frac{1-q}{1+q} \dot{q} / q\right]
$$

and

$$
\dot{a} / a=-\dot{E} / E+2 \dot{M} / M+\frac{1-q}{1+q} \dot{q} / q
$$

All of the terms on the right hand sides of both equations can be written in terms of the fractional accretion rate $\dot{M} / M$. If the specific angular momentum of the accreting material 
is $j_{\text {gas }}$, then $\dot{J} / J=\left[j_{\text {gas }} /(J / M)\right] \dot{M} / M$. We find it convenient to parameterize $j_{\text {gas }}$ in terms of the canonical Keplerian momentum via $j_{\text {gas }}=\mathcal{J} \sqrt{G M a}$, giving

$$
\frac{\dot{J}}{J}=\mathcal{J} \frac{(1+q)^{2}}{q} \frac{1}{\left(1-e^{2}\right)^{1 / 2}} \frac{\dot{M}}{M}
$$

¿From the MHD simulations of an equal-mass, circular retrograde binary in Bankert et al. (2014), we find $\mathcal{J} \approx-1.25$. Assuming the inner edge of the circumbinary disk is limited by the radius of the binary orbit at apocenter, we believe it is reasonable to scale this term with eccentricity as $\mathcal{J} \approx-1.25 \sqrt{1+e}$.

We parameterize the relative fraction of gas accreting onto each black hole as $f_{1}$ and $f_{2}$ $\left(f_{1}+f_{2}=1\right)$, giving an expression for $\dot{q}$ :

$$
\frac{\dot{q}}{q}=\frac{1+q}{q}\left(f_{2}-q f_{1}\right) \frac{\dot{M}}{M} .
$$

Here and in the following, quantities subscripted by 1 pertain to the primary, those subscripted by 2 pertain to the secondary.

The relation between $\dot{E}$ and $\dot{M}$ is harder to determine because it depends on how much dissipation is associated with attaching the orbital streams to the members of the binary. Because shocks are extremely likely, dissipation will in most instances be very significant. Here we choose to estimate the energy delivered by accretion on the basis that the system is maximally dissipative, that is, that shocks are capable of dissipating as much orbital energy into heat (and likely radiating it) as is consistent with momentum conservation. In other words, the gas streams inward from the circumbinary disk and then collides inelastically with one or the other of the "minidisks", the individual accretion disks around each black hole.

We assume that the accretion stream encounters the minidisk when the binary is at apocenter. Although we cannot confirm this assumption with our simulation data (the binary in the simulation had zero eccentricity), it is quite plausible: after all, this is the orbital phase at which the members of the binary are closest to the circumbinary disk, and also the phase at which the binary spends most of its time.

At apocenter, the total energy in the orbit can be written as

$$
\begin{aligned}
E & =-\frac{G M_{1} M_{2}}{2 a}=-\frac{G M^{2}}{2 a} \frac{q}{(1+q)^{2}} \\
& =-\frac{G M^{2}}{a(1+e)} \frac{q}{(1+q)^{2}}+\frac{1}{2} M_{1} v_{1}^{2}+\frac{1}{2} M_{2} v_{2}^{2},
\end{aligned}
$$


where in the second line we separate the energy into potential and kinetic terms, with $v_{1,2}$ the velocities at apocenter, and $r_{1,2}=a(1+e)(q, 1) /(1+q)$ the radial positions of the two black holes. Here we adopt a notation where the respective quantities for the two black holes are written $(1,2)$. The rate of change in the binary's potential energy is then

$$
\begin{aligned}
\dot{E}_{p}=-\frac{G\left(M_{2} \dot{M}_{1}+\dot{M}_{2} M_{1}\right)}{a(1+e)} & =-\frac{G M^{2}}{a(1+e)} \frac{\dot{M}}{M}\left(\frac{q}{1+q} f_{1}+\frac{1}{1+q} f_{2}\right) \\
& =2 E \frac{\dot{M}}{M} \frac{1}{1+e} \frac{1+q}{q}\left(q f_{1}+f_{2}\right) .
\end{aligned}
$$

For the kinetic energy terms, it is convenient to write the kinetic energy at apocenter in terms of the linear momentum of each $\mathrm{BH}: E_{k 1,2}=1 / 2\left(p_{1,2}^{2} / M_{1,2}\right)$. Then we have

$$
\dot{E}_{k 1,2}=\frac{p_{1,2} \dot{p}_{1,2}}{M_{1,2}}-\frac{p_{1,2}^{2}}{2 M_{1,2}^{2}} \dot{M} f_{1,2}=v_{1,2} \dot{p}_{1,2}-\frac{1}{2} v_{1,2}^{2} \dot{M} f_{1,2}
$$

where the linear velocities at apocenter are

$$
v_{1,2}=\sqrt{\frac{G M}{a}} \sqrt{\frac{1-e}{1+e}} \frac{(q, 1)}{1+q} \equiv v_{\text {apo }} \frac{(q, 1)}{1+q} .
$$

The momentum change comes from the accretion stream:

$$
\dot{p}_{1,2}=v_{\text {gas } 1,2} \dot{M} f_{1,2}
$$

which has velocity

$$
v_{\text {gas } 1,2}=\frac{j_{\text {gas }}}{r_{1,2}}
$$

As above, $j_{\text {gas }}$ is the specific angular momentum of the gas in the accretion stream. We can now write

$$
v_{\text {gas } 1,2}=\frac{\mathcal{J} \sqrt{G M a}}{a(1+e)} \frac{1+q}{(q, 1)}=\mathcal{J} \sqrt{\frac{G M}{a}} \sqrt{\frac{1-e}{1+e}} \sqrt{\frac{1}{1-e^{2}}} \frac{1+q}{(q, 1)}=\mathcal{J} v_{\text {apo }} \sqrt{\frac{1}{1-e^{2}}} \frac{1+q}{(q, 1)} .
$$

Now we can find the change in kinetic energy:

$$
\begin{aligned}
\dot{E}_{k 1,2} & =v_{\text {apo }}^{2} \sqrt{\frac{1}{1-e^{2}}} \mathcal{J} \dot{M} f_{1,2}-\frac{1}{2} v_{\text {apo }}^{2} \frac{q^{2}, 1}{(1+q)^{2}} \dot{M} f_{1,2} \\
& =-\dot{M} f_{1,2} v_{\text {apo }}^{2}\left[-\mathcal{J} \sqrt{\frac{1}{1-e^{2}}}+\frac{1}{2} \frac{\left(q^{2}, 1\right)}{(1+q)^{2}}\right] .
\end{aligned}
$$


Writing

$$
v_{\text {apo }}^{2}=\left(\frac{-2 E}{M}\right) \frac{1-e}{1+e} \frac{(1+q)^{2}}{q},
$$

and combining this with the expression for the potential energy, we get

$$
\frac{\dot{E}_{1,2}}{E}=\frac{\dot{M}}{M} f_{1,2} \frac{1}{1+e}\left[-2 \mathcal{J} \sqrt{\frac{1-e}{1+e}} \frac{(1+q)^{2}}{q}+(1-e)\left(q, q^{-1}\right)+2 \frac{1+q}{q}(q, 1)\right]
$$

Recall that, since $\mathcal{J}<0$, this expression is always strictly positive. Therefore the binary always evolves toward greater binding energy.

We can now inspect equations (2a) in a few limiting cases: $q=1, q \ll 1, e \ll 1$, $1-e=\delta \ll 1$. The leading-order terms in each case are shown in Table 1. For $q \ll 1$ we assume $f_{2}=1$. Not surprisingly, the eccentricity always grows and the semi-major axis always shrinks for binaries in the presence of retrograde accretion disks. Also not surprisingly, the effect is fastest when $q$ is small. Note that all evolution terms are proportional to $q^{-1} \dot{M} / M$, so the characteristic time scales can be rather shorter than the accretion time $M / \dot{M}=4.5 \times 10^{7} \dot{m}^{-1}$ yr, with $\dot{m}$ the Eddington-scaled accretion rate for a radiative efficiency of $\eta=0.1$.

Table 1: Leading-order terms for $\dot{e}$ and $\dot{a}$ for limiting values of $q$ and $e$.

\begin{tabular}{cccc}
$q$ & $e$ & $\dot{e}$ & $\dot{a} / a$ \\
\hline 1 & $\ll 1$ & $13 \frac{M}{M}$ & $-13 \frac{M}{M}$ \\
$\ll 1$ & $\ll 1$ & $\frac{9}{2 q} \frac{\dot{M}}{M}$ & $-\frac{9}{2 q} \frac{\dot{M}}{M}$ \\
1 & $1-\delta$ & $10 \sqrt{\delta} \frac{\dot{M}}{M}$ & $-5 \sqrt{\delta} \frac{\dot{M}}{M}$ \\
$\ll 1$ & $1-\delta$ & $\frac{2.5}{q} \sqrt{\delta} \frac{\dot{M}}{M}$ & $-\frac{1.25}{q} \sqrt{\delta} \frac{\dot{M}}{M}$
\end{tabular}

Because we assume that all accretion takes place at apocenter, and the accretion flow is tangent to the binary orbit at that point, the impulse approximation implies that $a(1+e)$ should remain constant. When $e \ll 1$, this implies that $\dot{e}=-\dot{a} / a$, while $\dot{e}=-2 \dot{a} / a$ when $e \approx 1$. This results are consistent with the limiting behavior shown in Table 1 .

\section{Gravitational Wave-Driven Evolution}

In the previous section, we saw that the binary eccentricity can grow rapidly via retrograde accretion. Nixon et al. (2011) also reached this conclusion, and predicted that, as 
eccentricity grew and the pericenter separation decreased, gravitational waves would take over as the driving evolutionary mechanism of the system. Here we present a more detailed analysis of this process. Peters (1964) gives the gravitational-wave-driven evolution of eccentricity and separation in the post-Newtonian regime:

$$
\begin{aligned}
(\dot{a} / a)_{\mathrm{GW}} & =-\frac{64}{5} \frac{q}{(1+q)^{2}}\left(1-e^{2}\right)^{-7 / 2}\left(1+\frac{73}{24} e^{2}+\frac{37}{96} e^{4}\right)\left(\frac{r_{g}}{a}\right)^{4} t_{g}^{-1} \\
(\dot{e})_{\mathrm{GW}} & =-\frac{304}{15} \frac{q}{(1+q)^{2}}\left(1-e^{2}\right)^{-5 / 2} e\left(1+\frac{121}{304} e^{2}\right)\left(\frac{r_{g}}{a}\right)^{4} t_{g}^{-1} .
\end{aligned}
$$

We have scaled the spatial dimensions by the gravitational radius $r_{g} \equiv G M / c^{2}$ and the time scale by $t_{g}=r_{g} / c$.

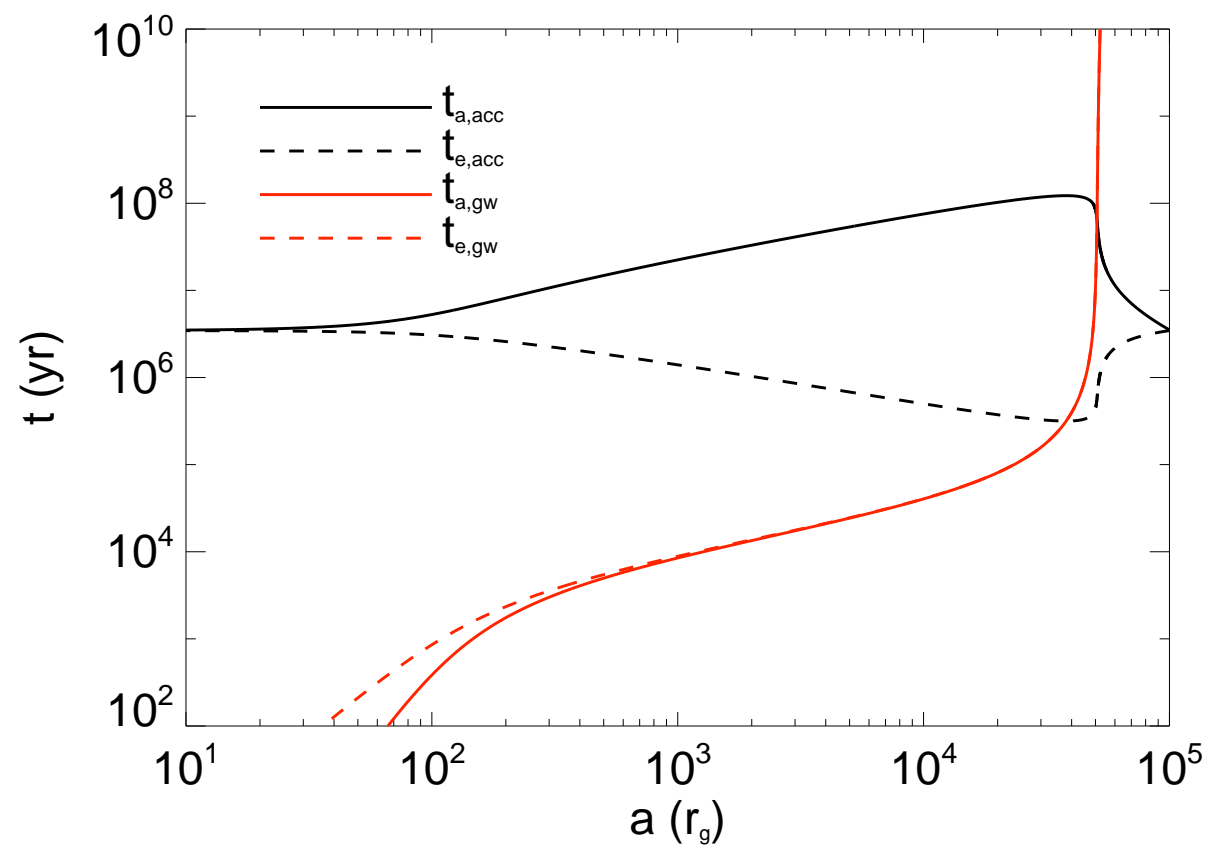

Fig. 1.- Characteristic time scales for various evolutionary processes. As the system evolves from right to left, first the eccentricity rises rapidly with constant $a(1+e)$ and then gravitational-wave-driven evolution takes over, decreasing both $a$ and $e$, while keeping $a(1-e)$ roughly constant. We take for initial values $M=10^{8} M_{\odot}, q=1, \dot{m}=1, a_{0}=10^{5} r_{g}$, and $e_{0}=0 . t_{\mathrm{e}, \mathrm{gw}}=t_{\mathrm{a}, \mathrm{gw}}$ over a broad range of semi-major axes because $a(1-e)$ is constant.

For a given $a, e$ we can now define the characteristic time scales $t_{e}=(1-e) / \dot{e}$ and $t_{a}=a / \dot{a}$ for the competing evolutionary processes: accretion and gravitational waves. For 
a fiducial binary system with initial parameters $M=10^{8} M_{\odot}, q=1, \dot{m}=1, a=10^{5} r_{g}$, and $e=0$, we plot in Figure 1 the relevant timescales as a function of $a(t)$. The graph should be read from right to left, as a shrinks with time. Whichever timescale is shortest at a given orbital separation is the dominant process governing the binary evolution at that point.

Consider, for example, the evolution of our fiducial case. As it accretes from its circumbinary disk, its eccentricity grows rapidly for a few million years, while its semi-major axis simultaneously shrinks by a factor of two. At this point, the high eccentricity leads to an extremely sharp decrease in $t_{a, \mathrm{gw}}$ and the system transitions from accretion-driven to gravitational wave-driven evolution. For highly eccentric orbits, most of the gravitational radiation is emitted at pericenter passage, when the two bodies have the highest velocity. This fact justifies an impulse approximation in which the energy and angular momentum are lost instantaneously at pericenter, so the binary evolves with constant $a(1-e)$.

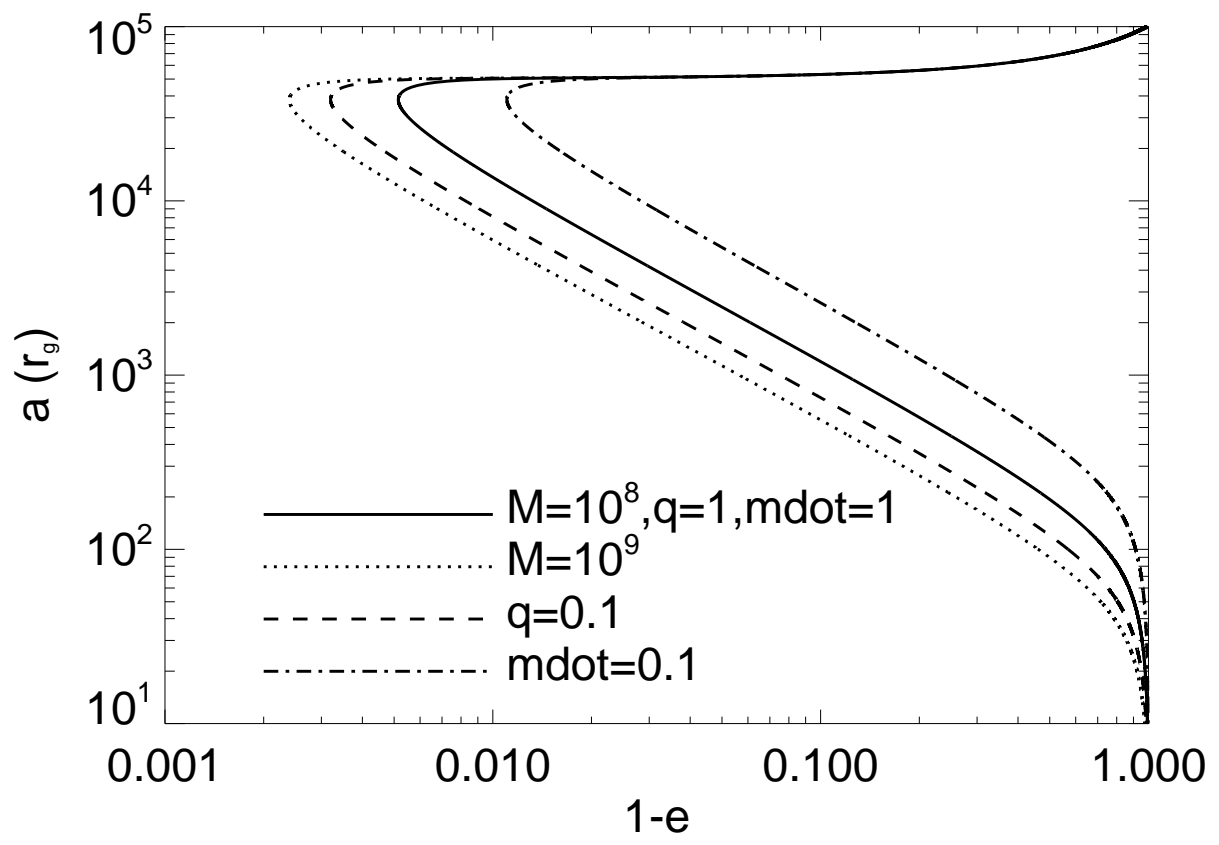

Fig. 2.- Evolution of $a$ and $e$ for the fiducial binary model with $M=10^{8} M_{\odot}, q=1$, and $\dot{m}=1$ (solid curve). We also show variations from this model with $M=10^{9} M_{\odot}$ (dotted curve), $q=0.1$ (dashed curve), and $\dot{m}=0.1$ (dot-dashed curve).

In Figure 2 we show the mutual evolution of $a$ and $e$ for a few variations of the fiducial model parameters. In all cases the binary begins in the upper-right corner with $a=10^{5} r_{g}$ and $e=0$. The dependence on the various parameters is straight-forward. Because $t_{g}$ grows with 
$M$, whereas for fixed $\dot{m}$ the accretion time is mass-independent, more massive systems reach a higher eccentricity before gravitational waves begin to dominate. For decreasing $\dot{m}$, the opposite happens: the accretion time increases even though the gravitational time remains constant, so the system leaves the accretion-dominated regime earlier, at a smaller eccentricity. For small $q$, the gravitational wave timescales increase and the accretion timescales decrease; both effects combine to push the system to higher eccentricity before entering the gravitational wave regime. More generally, because $a(1+e)$ is constant so long as the evolution is accretion-dominated, and the transition to GW-driven evolution occurs when $e \simeq 1$, the semimajor axis at this transition is always $\simeq 1 / 2$ the initial semimajor axis.

We can solve for the peak eccentricity $e_{*}$ by simply solving $\dot{e}_{\mathrm{acc}}=-\dot{e}_{\mathrm{GW}}$ in the limit of $1-e_{*}=\delta_{*} \ll 1$. For $\dot{e}_{\text {acc }}$ we use equations (2a) and (3) and the observation that the $\dot{J} / J$ terms dominate the evolution at high eccentricity. In this limit we find

$$
\delta_{*}=4 \times 10^{-3}\left[\frac{q^{2}}{(1+q)^{4}} a_{5}^{-4} M_{8}^{-1} \dot{m}^{-1}\right]^{1 / 3},
$$

which agrees quite well with the numerical evolutions plotted in Figure 2. Here we use the scaling expressions $a_{5}=a /\left(10^{5} r_{g}\right)$ and $M_{8}=M /\left(10^{8} M_{\odot}\right)$. For a wide range of parameters we find the time $t_{\text {trans }}$ needed to evolve from $e_{0}$ to $e_{*}$ can be approximated as

$$
t_{\text {trans }} \approx \frac{1}{3} \frac{q}{(1+q)^{2}}\left(1-e_{0}^{2}\right)^{1 / 2} \dot{m}^{-1} t_{\text {Sal }} .
$$

Because $q /(1+q)^{2}$, also called "the symmetric mass ratio", is always $\leq 1 / 4, t_{\text {trans }} \leq$ $(1 / 12)\left(t_{\text {Sal }} / \dot{m}\right)$, where $\left(t_{\text {Sal }} / \dot{m}\right)$ is the time over which accretion increases the mass of the system by one $e$-fold.

\section{Gravitational Wave Signal}

For binaries on circular orbits, the gravitational waveform is a sinusoidal signal with frequency exactly twice that of the orbital frequency $f_{\text {orb }}$. For non-zero eccentricities, the waveform is decidedly non-sinusoidal, as the instantaneous orbital frequency increases as the black holes move from apocenter to pericenter, peaking at

$$
f_{p}=f_{\text {orb }} \frac{(1+e)^{1 / 2}}{(1-e)^{3 / 2}} .
$$

Consequently, the range of harmonics carrying substantial power increases with increasing eccentricity (Tessmer \& Gopakumar 2007; Enoki \& Nagashima 2007). 
We calculate the amplitude $h(t)$ of the leading-order GW waveform from the timevarying quadrupole moment: $h(t) \sim \ddot{Q}$. The power emitted in the waveform is $L_{\mathrm{GW}}(t) \sim$ $\dot{h}^{2}(t)$. Taking the Fourier transform $L_{\mathrm{GW}}(f)$, we get the distribution of power in the individual harmonics. To get the correct normalization for $L_{\mathrm{GW}}(f)$, we use the orbit-averaged energy radiated $d E / d t$

$$
\frac{d E}{d t}=\frac{G M^{2} q}{(1+q)^{2}} \frac{\dot{a}}{2 a^{2}},
$$

where $\dot{a} / a$ is given by Peters (1964). We find that, for $1-e \ll 1$, the instantaneous $d E / d f$ peaks around $f_{p}$. For circular orbits $f_{\mathrm{GW}}=2 f_{p}$, so the fact that $f_{\mathrm{GW}} \approx f_{p}$ for eccentric orbits reflects the fact that the GW radiation is emitted over a finite part of the orbit around pericenter, during which $f<f_{p}$.

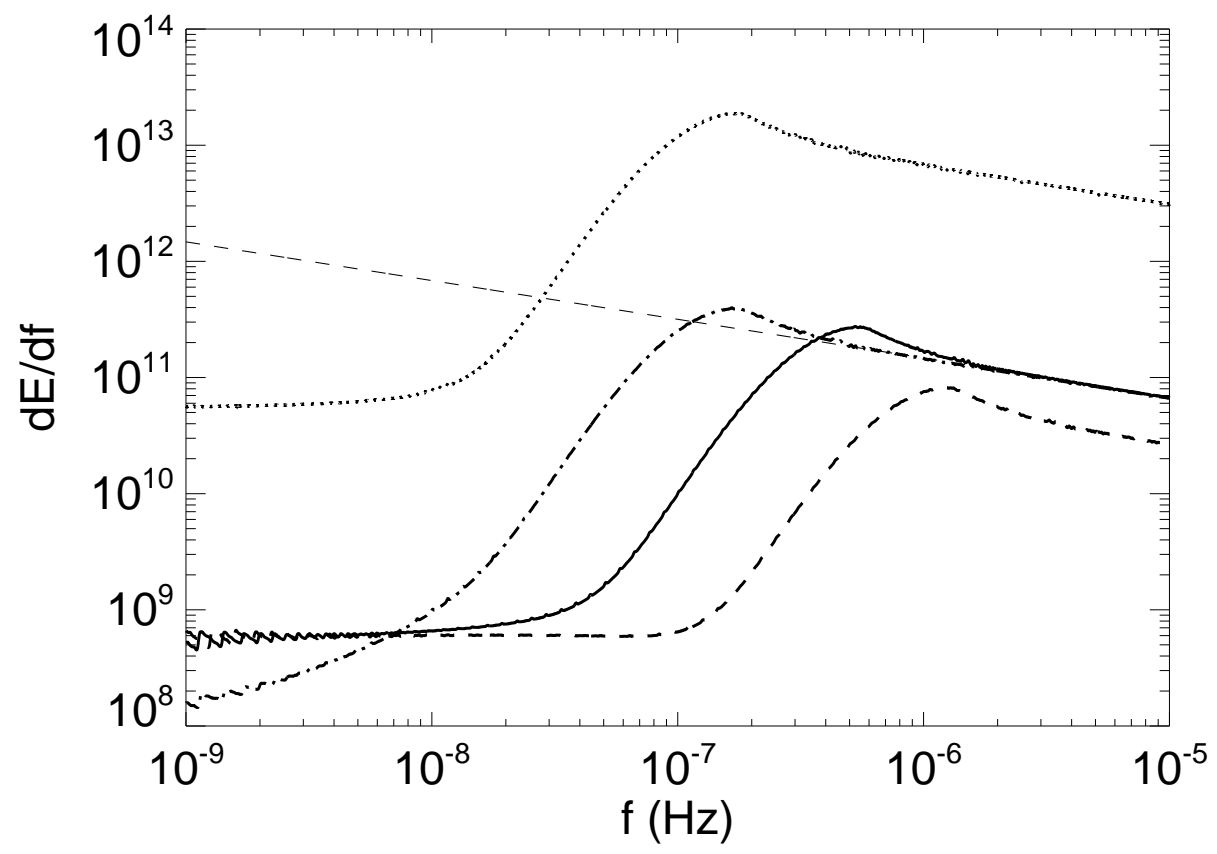

Fig. 3.- Gravitational-wave power spectrum for the fiducial binary model with $M=10^{8} M_{\odot}$, $q=1$, and $\dot{m}=1$ (solid curve). We also show variations from this model with $M=10^{9} M_{\odot}$ (dotted curve), $q=0.1$ (dashed curve), and $\dot{m}=0.1$ (dot-dashed curve). The thin dashed line is $d E / d f \sim f^{-1 / 3}$.

Figure 3 shows the GW power spectrum for the same four cases considered in Figure 2. fiducial model with $M=10^{8} M_{\odot}, q=1$, and $\dot{m}=1$ (solid curve), along with variations from this model with $M=10^{9} M_{\odot}$ (dotted curve), $q=0.1$ (dashed curve), and $\dot{m}=0.1$ 
(dot-dashed curve). The thin dashed line corresponds to $d E / d f \sim f^{-1 / 3}$, the classical result for purely circular evolution.

Figure 3 can best be understood by close comparison with Figure 2. As the binary's eccentricity increases through accretion, the GW signal rises sharply in amplitude and frequency until the binary evolution switches over to being dominated by gravitational waves. At this point, the binary evolves with constant $a(1-e)$, and thus constant $f_{\mathrm{GW}}$, building up power in a single peak. Eventually, the eccentricity is small enough that the system follows the standard circular evolution track, with both amplitude and frequency evolving to give $d E / d f \sim f^{-1 / 3}$.

Comparing it with Figure 2, we can also easily understand the relative offsets between the different models plotted in Figure 3. For the more massive system with $M=10^{9} M_{\odot}$ (dotted curve), the peak frequency shifts to the left since the orbital frequency decreases with increasing mass. At the same time, the GW amplitude increases because $d E / d f \propto M^{5 / 3}$. For smaller accretion rate ( $\dot{m}=0.1$; dot-dashed curve), the peak eccentricity is smaller, giving a lower peak frequency [see eqn. (18)], but it eventually evolves along exactly the same curve as the fiducial case. For smaller mass ratio $(q=0.1$; dashed curve), the peak eccentricity is larger, leading to a higher peak frequency. The amplitude scales like $d E / d f \propto q$, so the net effect is to shift the fiducial curve down and to the right.

The area under the solid black curve is actually the same as that under the thin dashed line because they both represent the total energy radiated going from $a \sim 10^{5} r_{g}$ down to merger. Thus, all the power normally radiated at low frequencies is concentrated in a small peak around $f \sim 10^{-7}-10^{-6} \mathrm{~Hz}$. This modification of the GW spectrum can potentially have a major impact on the stochastic gravitational wave signal being searched for by pulsar timing arrays (PTAs), which are most sensitive to GW signals in the $10^{-9}-10^{-8} \mathrm{~Hz}$ range (Hobbs et al. 2010; Shannon et al. 2013; Demorest et al. 2013; Sesana 2013).

If we take the initial separation to be somewhat smaller $\left(a=10^{4} r_{g}\right)$, we find qualitatively similar behavior in the evolution, but from equation (16) we see the peak eccentricity is "only" $e \approx 0.9-0.95$. In this case, the peaks in Figure 3 all shift to lower frequencies by a factor of $\sim 2$. Integrating over the cosmic population of SMBHBs, this shift could give a PTA stochastic signal with a peak around $f \approx 10^{-7} \mathrm{~Hz}$ and an amplitude roughly $50 \%$ higher than the canonical value, while sharply reducing the signal at lower frequencies. 


\section{Rapid Approach to Decoupling}

During the accretion-driven phase, the binary separation $a(1+e)$ remains constant, so the radius at which the secondary is farthest from the center-of-mass, and therefore best able to capture accreting gas, remains unchanged. Numerous studies (Roedig \& Sesana 2014; D'Orazio et al. 2013; Farris et al. 2014b; Shi \& Krolik 2015) have found that so long as $q$ is not too small, the secondary captures the majority of the accretion flow from a surrounding disk in prograde binaries; that fact is likely to carry over to the retrograde case. On the other hand, during the GW-driven phase, $r_{p}=a(1-e)$ is fixed, while the apocenter evolves like $r_{a}=r_{p}(1+e) /(1-e)$, which falls from $\simeq 2 a_{*}$ to $\simeq a_{*}\left(1-e_{*}\right)$ as the orbit grows less eccentric ( $a_{*}$ and $e_{*}$ are the semi-major axis and eccentricity at the point when the GW-driven phase begins).

The rapid diminution in apocenter distance during the GW-driven phase may be faster than the characteristic inflow rate through the inner region of the accretion disk. If during the relevant phases of orbital evolution, the disk is in the Shakura-Sunyaev "outer zone" (gas pressure-dominated, free-free opacity greater than electron scattering opacity), this inflow time at radius $r=r_{5} /\left(10^{5} r_{g}\right)$ is

$$
t_{\text {inflow }} \approx 7 \times 10^{8} \dot{m}^{-3 / 10} \alpha_{-1}^{-4 / 5} M_{8}^{6 / 5} r_{5}^{7 / 5} \mathrm{yr} .
$$

Here $\alpha=10^{-1} \alpha_{-1}$ is the parameter defined by Shakura \& Sunvaev (1973) to describe the ratio of the vertically-integrated internal stress to the vertically-integrated pressure, scaled to its canonical value of 0.1 . While $t_{\text {inflow }}$ is somewhat larger than both $t_{a \text {,acc }}$ and $t_{e, \text { acc }}$ during the accretion-dominated phase (see Fig. 1), this does not actually imply decoupling, since the important quantity for comparison is the apocenter evolution timescale, which is essentially infinite at this phase in the evolution, so the disk is likely in inflow equilibrium.

Equation (20) assumes a steady-state thin disk geometry, in which the local temperature is controlled by a balance between heating due to accretion and local radiation. However, it has been recognized for many years that for typical AGN parameters, such disks are unstable to self-gravity at $r \gtrsim 10^{3} r_{g}$ (Shlosman \& Begelman 1987; Goodman 2003; Jiang \& Goodman 2011; Dunhill et al. 2014), and this range of radii is of particular interest to the questions studied here. Unfortunately, little is known about what actually happens as a result. It is possible, as these papers have discussed, that the disk fragments and a significant part of its mass is concentrated into new stars.

On the other hand, we have observational evidence at larger scales $\left(\gtrsim 10^{5} r_{g}\right)$ that accreting material forms geometrically thick structures, so-called "obscuring tori;" most recent work has supposed that they are supported by the AGN's radiation pressure interacting with

dust grains in the gas (Pier \& Krolik 1992; Chang et al. 2007; Krolik 2007; Dorodnitsyn et al. 
2011, 2012; Roth et al. 2012). To account for additional mechanisms that might alter the disk's vertical support, it is useful to rephrase the inflow time in terms of the disk aspect ratio $h / r$, its ratio of vertical scale height to radial position. The inflow time then becomes

$$
t_{\text {inflow }}=5 \times 10^{7} \alpha_{-1}^{-1} M_{8} r_{5}^{3 / 2}\left(\frac{h / r}{0.01}\right)^{-2} \mathrm{yr} .
$$

We scale $h / r$ in this instance to $10^{-2}$ because the aspect ratio predicted by classical disk theory is $2 \times 10^{-3} \alpha^{-1 / 10} \dot{m}^{3 / 20} M_{8}^{-1 / 4} r_{5}^{1 / 20}$, and this is presumably a lower bound.

By comparison, the characteristic gravitational wave timescale $t_{\mathrm{GW}}=a / \dot{a}$ in the limit of $e \rightarrow 1$ is

$$
t_{\mathrm{GW}}=5 \times 10^{5} \frac{(1+q)^{2}}{q} M_{8} a_{5}^{4}\left(\frac{\delta_{*}}{0.003}\right)^{7 / 2} \mathrm{yr} .
$$

We have scaled to the typical orbital elements when gravitational wave evolution begins to dominate. We therefore expect that in most instances, the apocenter changes too rapidly for the bulk of the disk to follow once GW emission controls the system's evolution.

Milosavljević \& Phinney (2005) argued that whenever the binary evolution does become faster than the disk's inflow time, mass inflow is cut off, "decoupling" the disk from the binary and ending electromagnetic emission due to accretion. On the other hand, the global MHD simulation of Noble et al. (2012) showed that the accretion rate can, in fact, be sustained even in this regime - edge effects can substantially raise the ratio of stress to pressure above its usual value. Similar effects are generically seen during the transient phases of almost all global MHD accretion simulations (Hawley et al. 2011; Sorathia et al. 2012). In fact, analogous edge effects occur even in purely hydrodynamic treatments (Farris et al. 2014a). Thus, the degree to which mass accretion actually diminishes when the orbital evolution time is shorter than the disk inflow time is uncertain. In either case, retrograde disks should reach this "decoupling" considerably sooner than prograde disks.

\section{Disk-Binary Alignment}

A binary and its disk can remain in a stable counter-aligned orientation if $J_{\text {disk }}<2 J_{\text {bin }}$ (Nixon 2012). However, this criterion is difficult to satisfy in the long-run. The relevant disk mass is the total amount of mass passing through it integrated over time, and, as the binary's eccentricity approaches unity, $J_{\text {bin }}$ falls. Both effects make it easier to flip the binary

around while still conserving the total angular momentum of the system (Roedig \& Sesana 2014). We have shown already that the angular momentum conveyed with mass transfer is insufficient to accomplish this transition to a prograde orientation before gravitational 
wave emission brings the binary to merger; however, the situation changes when the binary orbital plane and the disk plane are not exactly co-planar because in that configuration angular momentum can also be transferred between the two systems via the mutual torque arising from the binary's quadrupole moment.

Suppose, then, that the angular momentum fed to the disk at large radius is aligned not exactly opposite to the angular momentum of the binary, but only approximately. As shown by Miller \& Krolik (2013), when the binary orbit is circular, the inner portion of the disk aligns relatively quickly with the orbital plane, but beyond a certain distance the disk maintains its intrinsic orientation. Once the disk has reached a steady-state shape, the rate at which angular momentum is delivered from the disk to the binary is $\sim \dot{M} j_{T}$, where $j_{T}$ is the magnitude of the misaligned specific angular momentum in the disk at the radius where the disk orientation transitions from aligned to misaligned, and the radius of that transition is $R_{T} \sim(r / h)_{T} a$, where $(h / r)_{T}$ is the disk aspect ratio at $R_{T}$.

For the present application, this result must be generalized in two ways. The first is that their work assumed the binary has a circular orbit, so it must be extended to eccentric orbits. Following Landau \& Lifshitz (1969), it is easy to show that the time-averaged quadrupole moments of an eccentric binary are

$$
\begin{aligned}
Q_{x x} & =\frac{M}{2} \frac{q}{(1+q)^{2}} a^{2}\left(1+4 e^{2}\right) \\
Q_{y y} & =\frac{M}{2} \frac{q}{(1+q)^{2}} a^{2}\left(1-e^{2}\right),
\end{aligned}
$$

where we define the $x$ - and $y$ - directions as parallel to the major and minor axes of the ellipse, respectively. If the disk is tilted in the $\hat{e}-\hat{z}$ plane, where $\hat{z} \perp \hat{x}, \hat{y}$ and $\hat{e}=$ $\cos \phi \hat{x}+\sin \phi \hat{y}$, the torque is proportional to $Q_{x x} \cos ^{2} \phi+Q_{y y} \sin ^{2} \phi$.

Because the quadrupolar torque is precessional, both the orientation of the disk tilt plane $\phi$ and the misalignment angle $\theta$ can be functions of radius within the disk. The total binary-disk torque is then

$$
\begin{aligned}
\mathcal{T}=-\frac{3 \pi}{2} \frac{q}{(1+q)^{2}} \Omega_{T}^{2} a^{2} \sin \theta_{0} R_{T}^{2} \Sigma_{T} \times & \\
& \int d x x^{-2} \frac{\sin \theta(x)}{\sin \theta_{0}} \frac{\Sigma(x)}{\Sigma_{T}}\left[\left(1+4 e^{2}\right) \cos ^{2} \phi(x)+\left(1-e^{2}\right) \sin ^{2} \phi(x)\right],
\end{aligned}
$$

where quantities with subscript $T$ are evaluated at $R_{T}$, beyond which the disk's intrinsic alignment makes an angle $\theta_{0}$ with the binary. The orbital frequency at this location is $\Omega_{T}$, $\Sigma_{T}$ the surface density, and $x=r / R_{T}$. The form of this expression for $\mathcal{T}$ is written so as to make the integral both dimensionless and order unity, as in Miller \& Krolik (2013). 
Following their notation, we designate it as $\mathcal{I}$, but now recognize that, unlike in the case of circular binary orbits, it is a function of $e$ and precession phase profile $\phi(r)$.

The second generalization is a consequence of the implicit assumption in Miller \& Krolik (2013) that alignment of the binary remains incomplete even after the alignment front has propagated all the way through the inner disk, so that the torque is concentrated near $r \simeq R_{T}$. If, when the system is first formed, the misaligned angular momentum contained in the disk within a radius smaller than $R_{T}$ (the steady-state alignment transition radius) is comparable to the angular momentum of the binary, the binary will have reoriented before the alignment front arrives at the steady-state radius.

In fact, just this situation may often occur in the context of binary black holes. Suppose that the circumbinary disk extends from an inner radius $\simeq a$ (because it is obliquely retrograde) out to an outer radius $r_{\text {out }}$. As we did in the previous section when discussing the approach to decoupling, we will describe the disk's surface density profile in terms of a particular aspect ratio $h / r$, rather than calculate the aspect ratio from a model for the disk's thermal state. The ratio between its misaligned angular momentum and the binary's total angular momentum is then

$$
\frac{J_{\text {disk }}}{J_{\text {bin }}}=2 \pi \sin \theta_{0} \frac{\dot{m}}{\alpha \eta}\left(a / r_{g}\right)^{3 / 2}(r / h)^{2} \frac{\left[\left(r_{\text {out }} / a\right)^{2}-1\right](1+q)^{2}}{q\left(1-e^{2}\right)^{1 / 2}} \frac{G r_{g}}{\kappa_{T} c^{2}},
$$

where $\kappa_{T}$ is the Thomson opacity. If the scale height varies as a function of radius, $(r / h)^{2}$ should be regarded as the angular momentum-weighted mean of $(r / h)^{2}$. Evaluated for our fiducial values, this ratio is

$$
\frac{J_{\text {disk }}}{J_{\text {bin }}} \simeq 0.06 \sin \theta_{0}\left(\frac{\alpha}{0.1}\right)^{-1} \frac{\dot{m}}{\eta} M_{8}\left(\frac{a}{10^{5} r_{g}}\right)^{3 / 2}\left(\frac{r / h}{100}\right)^{2} \frac{1}{\left(1-e^{2}\right)^{1 / 2}} \frac{(1+q)^{2}}{q}\left[\left(r_{\text {out }} / a\right)^{2}-1\right] .
$$

In other words, for our fiducial values, the disk does not need to extend to a large multiple of $a$ in order for its angular momentum content to match the binary's. However, it is also clear that this ratio is sensitive to a variety of parameters.

Suppose first that $J_{\text {disk }} \gg J_{\text {bin. }}$. In this case, as the alignment front propagates out through the disk, the corresponding torque on the binary causes its angular momentum to swing toward the direction of the disk's angular momentum. Because the direction of the binary's angular momentum is initially nearly opposite to that of the outer disk, this swing initially increases the angle between the two orbital planes, the inclination angle growing exponentially if it is initially small (Scheuer \& Feiler 1996) 1 .

1 Nixon (2012) claimed otherwise, but he assumed a disk without continuing accretion supply and ran for too short a time to test stability. 
However, equation (26) suggests that there could also be disks of limited radial extent containing less misaligned angular momentum than the binary, and the maximum $r_{\text {out }} / a$ for which $J_{\text {disk }}<J_{\text {bin }}$ depends on the accretion rate, binary mass, etc. When $r_{\text {out }}$ is small enough for $J_{\text {disk }}<J_{\text {bin }}$, the disk counter-aligns swiftly, i.e., it moves into the binary orbital plane, but orbiting retrograde to the binary motion. Adapting the formalism of Sorathia et al. (2013) from the Lense-Thirring problem they considered to the closely analogous problem of alignment with a binary, we find that the alignment front moves outward at a rate

$$
v_{f}(r) \simeq \frac{3}{8} \frac{q}{(1+q)^{2}} \frac{a^{2} \Omega(r)}{r} \mathcal{I}(r) .
$$

In this expression $\mathcal{I}$ is redefined so that it covers the region within $r$ rather than within $R_{T}$. Consequently $x$ in equation (24) becomes $r^{\prime} / r$ and $\Sigma_{T}$ becomes $\Sigma(r)$. Thus, for order unity mass ratio in the binary, $v_{f} \sim(a / r)^{2} v_{\text {orb }}(r)$, not much slower than the orbital speed, and the time for alignment to be achieved throughout a truncated disk is $\sim\left(r_{\text {out }} / a\right)^{2}$ binary orbital periods at its outer radius.

Once such a disk is entirely aligned, the binary exerts no further torque on the disk proper, but does continue to interact with material at and beyond its outer boundary, $r_{\text {out }}$. Because the torque decreases rapidly with increasing radius (the precession frequency is $\propto$ $r^{-7 / 2}$ ), only the nearest matter is significant. Typically the mass just outside the disk should be much smaller than the mass within the disk because the surface density of the disk is $\sim$ $200(\alpha / 0.1)^{-1}(\dot{m} / \eta)[(r / h) / 100]^{2}(r / 10 a)^{-1 / 2}\left(a / 10^{5} r_{g}\right)^{-1 / 2} \kappa_{T}^{-1}$, a surface density much greater than would be expected from the surrounding interstellar medium. Consequently, the torque that the binary continues to exert is on the material that has just become attached to the disk, arriving with the intrinsic orientation of the mass supply: $\mathcal{T}=\sin \theta_{0} \dot{M} r_{\text {out }}^{2} \Omega\left(r_{\text {out }}\right)$. This torque is smaller than the steady-state torque estimated through the formalism of Miller \& Krolik (2013) by the ratio $\left(r_{\text {out }} / R_{T}\right)^{1 / 2}$.

Whether the binary evolves to gravitational wave-driven merger faster than it aligns with the external mass supply depends on whether $t_{\text {align }}$, the time required for this torque to change the orientation of the binary, is larger or smaller than $t_{\text {trans }}$, the time for accretion to drive binary orbital evolution. Because both timescales depend on the angular momentum content of the binary and the mass accretion rate, their ratio is very simple:

$$
\frac{t_{\text {trans }}}{t_{\text {align }}}=\frac{1}{3} \sin \theta_{0}\left(r_{\text {out }} / a\right)^{1 / 2} \text {. }
$$

Thus, the maximum size of the circumbinary disk permitting retrograde orbital evolution to reach its conclusion is limited by two constraints. One is that the timescale for eccentricity growth must be shorter than that for disk-binary alignment:

$$
r_{\text {out }} \lesssim\left(9 / \sin ^{2} \theta_{0}\right) a
$$


For moderate obliquity, $r_{\text {out }} / a$ could be as large as $\simeq 10-100$ and still satisfy this condition. The other constraint is the one described above in equation (26), namely that $J_{\text {disk }}$ be $\lesssim J_{\text {bin }}$. This requirement depends on more parameters. For our fiducial choices, it gives a tighter constraint, $r_{\text {out }} \lesssim 2 a / \sqrt{\sin \theta_{0}}$, but this constraint becomes similar to or looser than the timescale condition when $(\dot{m} / \eta) M_{8}\left(a / 10^{5} r_{g}\right)^{3 / 2}[(r / h) / 100]^{2}(1+q)^{2} / q \lesssim 0.1$ (e.g., smaller accretion rates, more compact binaries, or thicker outer disks).

It is worth pointing out that the timescale criterion we have used can be rephrased in terms of the total angular momentum and mass budgets. King et al. (2005) argued that the binary can be flipped when $J_{\text {disk }} / J_{\text {bin }}>2$. This criterion applies when there is no resupply of mass to the disk. The alignment timescale in our case should therefore be posed in terms of the ratio of the binary angular momentum to the rate at which (misaligned) angular momentum is supplied to the disk. The transition time $t_{\text {trans }}$ is defined above in equation (17), and is roughly the time it takes to accrete $20 \%$ of the secondary's mass.

Up to this point, we have ignored the question of whether such truncated disks might be expected in Nature. In fact, they may be reasonably common. Presumably what defines the outer edge of the circumbinary disk is the point at which interstellar gas, following ballistic orbits in the gravitational potential created by the binary black hole and the host galaxy, shocks against the disk's outer edge and joins the accretion flow. Another reason why the inventory of mass immediately outside the disk should be much smaller than the disk mass is, in fact, that mass in this region travels inward ballistically, whereas within the disk inward motion is dependent upon the comparatively slow process of internal angular momentum transport. The place where external matter joins the disk could be at a radius as small as that for which the specific angular momentum of the interstellar gas supports a roughly circular orbit; alternatively, it could be at larger radius if the accretion flow (having this orientation) has lasted long enough for the outer edge of the disk to be pushed outward significantly by the outward angular momentum flux of accretion.

Details of the formation of binary black holes are complex and depend upon numerous parameters of the galaxy mergers preceding the formation of such a binary. However, chaotic motion of interstellar gas on the scale of parsecs to tens of parsecs with orbital velocities of several hundred $\mathrm{km} / \mathrm{s}$ can often be seen in simulations of specific scenarios (Mayer et al. 2007; Roškar et al. 2014). By comparison, the physical scale of the of the disks we have been considering is similar or somewhat smaller, $\sim 5$ pc for our fiducial parameters with $r_{\text {out }} \sim 10 a$. Thus, a truncated obliquely retrograde disk of the sort we have discussed may well be formed. 


\section{Discussion}

To form a circumbinary accretion disk, one requires a SMBH binary and a supply of gas. Galactic mergers are almost certainly required to provide the binary, but although mergers may enhance the availability of gas, they are not strictly necessary, as we see plenty of examples of AGN in non-merging galaxies. Assuming a merger did take place and a SMBH binary has formed, if subsequent nuclear activity is driven by the stochastic accretion of molecular clouds with random orientation relative to the central SMBHB orbit, we expect it would be equally likely for circumbinary accretion disks to form in the prograde or retrograde orientations.

With major mergers happening every $\sim 3-10$ Gyr per galaxy in the local universe (Lotz et al. 2011), and typical AGN lifetimes $\sim 10^{7}$ years (Martini 2004) with duty cycle $~$ $10^{-2}$ (Marconi et al. 2004), we should expect multiple accretion phases between mergers. For prograde circumbinary disks, doubling the secondary mass leads to the binary orbit shrinking by a factor of order unity (Shi et al. 2012), so many prograde episodes would be necessary to reach the GW-dominated phase. However, retrograde disks lead to much more rapid orbital evolution: a mere $20 \%$ increase in the mass of the secondary suffices. For the same accretion rate at large radius, retrograde disks are more efficient in driving binary orbital evolution than prograde disks not because they transmit more mass to the binary [both cases maintain accretion flows close to inflow equilibrium (Farris et al. 2014b; Shi \& Krolik 2015)], but because retrograde accretion changes the specific angular momentum of the binary by a much larger factor. The angular momentum carried to the binary by accretion in the prograde case can in fact significantly counteract the angular momentum lost by the binary through gravitational torques on the circumbinary disk (Shi et al. 2012).

Thus, within this paradigm of multiple, stochastic accretion episodes between mergers, there is a strong statistical bias toward such rapid eccentricity evolution via accretion from retrograde disks that the GW-driven state is achieved quickly. When the mass accreted during a single episode of constant angular momentum orientation is less than roughly half the secondary mass, substantial orbital evolution occurs only for roughly retrograde orientation. Moreover, when substantial orbital evolution does take place due to a retrograde accretion episode, it drives the binary toward merger, so that no further episodes, retrograde or prograde, occur.

Torques couple binaries to obliquely-oriented circumbinary disks. When $\vec{J}_{\text {disk }} \cdot \vec{J}_{\text {binary }}<0$ and enough mass is available (either in the initial disk or over the course of its feeding) that the total disk angular momentum is larger than the binary's, these torques can "flip" the binary orbit so that it aligns with the disk plane and orbits in the same sense (Scheuer \& Feiler 1996; King et al. 2005). However, we have shown that if the disk is fed at radii not too much 
larger than the binary semimajor axis, the angular momentum instantaneously stored in the disk can be small enough, and the evolution of the binary orbit caused by retrograde accretion can be rapid enough, that the binary is driven to the gravitational wave-dominated stage before its orbit is flipped. Thus, the condition for retrograde accretion to cause merger before the binary orbit is reoriented amounts to a condition on the disk's outer radius relative to the scale of the binary orbit.

As a result, high-eccentricity and ultimately GW-driven SMBHBs may be a common result of gas-driven black hole mergers unless any of three conditions applies: accretion episodes frequently occur in which the total mass accreted is large enough to cause substantial binary orbital evolution even if the rotation sense is prograde; the orientation of the accreted matter's angular momentum is consistently correlated with that of the binary black hole system; or the outer edge of the circumbinary disk is so far outside the binary that retrograde systems are flipped before their orbits evolve. In evaluating this statement, we emphasize the qualifier "gas-driven" because gas accretion may not always be the principal element in binary black hole evolution: stellar interactions may be more important in some cases. This is especially relevant for the massive gas-poor elliptical galaxies that host the most massive black holes, and which are thought to dominate the PTA signal. These galaxies also exhibit decreased nuclear activity. It is also possible that even when stellar interactions are weak, the evolution of the circumbinary disk at large radii may be complicated by self-gravity and internal star formation.

One consequence of the gas-driven, rapid merger scenario would be a distinct absence of binary quasars with separations between $\sim 0.01-1 \mathrm{pc}$, a source population that has been notoriously difficult to detect (Eracleous et al. 2012). To the degree that gas-driven accretion dominates binary evolution in PTA sources, the effects we have pointed out would have profound effects on the potentially-observable GW signal, effectively moving most of the GW power from the $\mathrm{nHz}$ to the $\mu \mathrm{Hz}$ band (Sesana 2013). By the time such systems reach the $\mathrm{mHz}$ band, they should be highly circularized.

Because the GW evolution time drops so rapidly with increasing eccentricity, it is likely that at some point before black hole merger, the circumbinary disk will no longer be able to stretch inward as rapidly as the binary apocenter distance shrinks; that is, the the system will "decouple." Yet even in such a situation, there still might be enough low-density, highly magnetized gas in the central gap to lead to an observable EM signal. Understanding the precise nature of such a system will require new 3D global MHD simulations that can sufficiently resolve both the disk and the gas flow onto the individual black holes over long periods of time. 


\section{Acknowledgments}

This work was partially supported by National Science Foundation grant AST-1028111 and NASA grant ATP12-0139. We thank Cole Miller for helpful comments and discussion. We also thank the referee for leading us to pursue the properties of binary-circumbinary disk alignment much further than we had initially.

\section{REFERENCES}

Armitage, P. J., \& Natarajan, P. 2002, ApJL, 567, L9

Bankert, J., Krolik, J., \& Shi, J.-M. 2014, submitted to ApJ

Begelman, M. C., Blandford, R. D., \& Rees, M. J. 1980, Nature, 287, 307

Bell, E. F., Phleps, S., Somerville, R. S., et al. 2006, ApJ, 652, 270

Bridge, C. R., Carlberg, R. G., \& Sullivan, M. 2010, ApJ, 709, 1067

Chang, P., Quataert, E., \& Murray, N. 2007, ApJ, 662, 94

Chang, P., Strubbe, L. E., Menou, K., \& Quataert, E. 2010, MNRAS, 407, 2007

Cuadra, J., Armitage, P. J., Alexander, R. D., \& Begelman, M. C. 2009, MNRAS, 393, 1423

de Ravel, L., Le Fèvre, O., Tresse, L., et al. 2009, AAP, 498, 379

Demorest, P. B., Ferdman, R. D., Gonzalez, M. E., et al. 2013, ApJ, 762, 94

D’Orazio, D. J., Haiman, Z., \& MacFadyen, A. 2013, MNRAS, 436, 2997

Dorodnitsyn, A., Bisnovatyi-Kogan, G. S., \& Kallman, T. 2011, ApJ, 741, 29

Dorodnitsyn, A., Kallman, T., \& Bisnovatyi-Kogan, G. S. 2012, ApJ, 747, 8

Dunhill, A. C., Alexander, R. D., Nixon, C. J., \& King, A. R. 2014, MNRAS, 445, 2285

Enoki, M., \& Nagashima, M. 2007, Progress of Theoretical Physics, 117, 241

Eracleous, M., Boroson, T. A., Halpern, J. P., \& Liu, J. 2012, ApJS, 201, 23

Escala, A., Larson, R. B., Coppi, P. S., \& Mardones, D. 2004, ApJ, 607, 765

—. 2005, ApJ, 630, 152 
Farris, B. D., Duffell, P., MacFadyen, A. I., \& Haiman, Z. 2014a, ArXiv e-prints, arXiv:1409.5124

-. 2014b, ApJ, 783, 134

Goodman, J. 2003, MNRAS, 339, 937

Gould, A., \& Rix, H.-W. 2000, ApJL, 532, L29

Hawley, J. F., Guan, X., \& Krolik, J. H. 2011, ApJ, 738, 84

Hobbs, G., Archibald, A., Arzoumanian, Z., et al. 2010, Classical and Quantum Gravity, 27, 084013

Jiang, Y.-F., \& Goodman, J. 2011, ApJ, 730, 45

King, A. R., Lubow, S. H., Ogilvie, G. I., \& Pringle, J. E. 2005, MNRAS, 363, 49

Kocsis, B., \& Sesana, A. 2011, MNRAS, 411, 1467

Kormendy, J., \& Richstone, D. 1995, ARA\&A, 33, 581

Krolik, J. H. 2007, ApJ, 661, 52

Landau, L. D., \& Lifshitz, E. M. 1969, Mechanics

Lotz, J. M., Jonsson, P., Cox, T. J., et al. 2011, ApJ, 742, 103

MacFadyen, A. I., \& Milosavljević, M. 2008, ApJ, 672, 83

Magorrian, J., Tremaine, S., Richstone, D., et al. 1998, AJ, 115, 2285

Marconi, A., Risaliti, G., Gilli, R., et al. 2004, MNRAS, 351, 169

Martini, P. 2004, Coevolution of Black Holes and Galaxies, 169

Mayer, L., Kazantzidis, S., Madau, P., et al. 2007, Science, 316, 1874

McIntosh, D. H., Guo, Y., Hertzberg, J., et al. 2008, MNRAS, 388, 1537

Merritt, D., \& Milosavljević, M. 2005, Living Reviews in Relativity, 8, 8

Miller, M. C., \& Krolik, J. H. 2013, ApJ, 774, 43

Milosavljević, M., \& Phinney, E. S. 2005, ApJL, 622, L93

Nixon, C. J. 2012, MNRAS, 423, 2597 
Nixon, C. J., Cossins, P. J., King, A. R., \& Pringle, J. E. 2011, MNRAS, 412, 1591

Noble, S. C., Mundim, B. C., Nakano, H., et al. 2012, ApJ, 755, 51

Peters, P. C. 1964, Physical Review, 136, 1224

Pier, E. A., \& Krolik, J. H. 1992, ApJL, 399, L23

Ravi, V., Wyithe, J. S. B., Shannon, R. M., Hobbs, G., \& Manchester, R. N. 2014, MNRAS, 442,56

Roedig, C., \& Sesana, A. 2014, MNRAS, 439, 3476

Roth, N., Kasen, D., Hopkins, P. F., \& Quataert, E. 2012, ApJ, 759, 36

Roškar, R., Mayer, L., Fiacconi, D., et al. 2014, ArXiv e-prints, arXiv:1406.4505

Scheuer, P. A. G., \& Feiler, R. 1996, MNRAS, 282, 291

Schnittman, J. D. 2013, Classical and Quantum Gravity, 30, 244007

—. 2014, ArXiv e-prints, arXiv:1411.3994

Sesana, A. 2013, Classical and Quantum Gravity, 30, 224014

Shakura, N. I., \& Sunyaev, R. A. 1973, AAP, 24, 337

Shannon, R. M., Ravi, V., Coles, W. A., et al. 2013, Science, 342, 334

Shi, J.-M., \& Krolik, J. H. 2015, in preparation

Shi, J.-M., Krolik, J. H., Lubow, S. H., \& Hawley, J. F. 2012, ApJ, 749, 118

Shlosman, I., \& Begelman, M. C. 1987, Nature, 329, 810

Sorathia, K. A., Krolik, J. H., \& Hawley, J. F. 2013, ApJ, 777, 21

Sorathia, K. A., Reynolds, C. S., Stone, J. M., \& Beckwith, K. 2012, ApJ, 749, 189

Tanaka, T., \& Menou, K. 2010, ApJ, 714, 404

Tessmer, M., \& Gopakumar, A. 2007, MNRAS, 374, 721 